\title{
IL-17 and TNF- $\alpha$ Are Key Mediators of Moraxella catarrhalis Triggered Exacerbation of Allergic Airway Inflammation
}

\author{
Safa Alnahas', Stefanie Hagner' ${ }^{2}$, Hartmann Raifer ${ }^{1}$, Ayse Kilic ${ }^{2}$, \\ Georg Gasteiger ${ }^{3}$, Reinier Mutters' ${ }^{1}$, Anne Hellhund ${ }^{1}$, Immo Prinz', \\ Olaf Pinkenburg', Alexander Visekruna', Holger Garn ${ }^{2}$ and Ulrich Steinhoff' *
}

1/nstitute of Medical Microbiology and Hospital Hygiene, University of Marburg, Marburg, Germany, 2Institute of Laboratory Medicine and Pathobiochemistry, Molecular Diagnostics, Member of the German Center for Lung Research, University of Marburg, Marburg, Germany, ${ }^{3}$ Institute of Medical Microbiology and Hygiene, FZI Research Center for Immunotherapy, University of Mainz Medical Center, Mainz, Germany, ${ }^{4}$ Institute of Immunology, Hannover Medical School, Hannover, Germany

OPEN ACCESS

Edited by:

Tobias Schuerholz, Universitätsmedizin Rostock,

Germany

Reviewed by:

Barbara M. Bröker,

University of Greifswald, Germany

Esmaeil Mortaz,

Utrecht Institute for Pharmaceutical

Sciences, Netherlands

*Correspondence:

Ulrich Steinhoff ulrich.steinhoff@staff.uni-marburg.de

Specialty section: This article was submitted to Inflammation,

a section of the journal

Frontiers in Immunology

Received: 03 April 2017 Accepted: 31 October 2017 Published: 14 November 2017

Citation:

Alnahas S, Hagner S, Raifer H, Kilic A, Gasteiger G, Mutters R, Hellhund A, Prinz I, Pinkenburg O, Visekruna A, Garn $H$ and Steinhoff $U$ (2017) IL-17 and TNF- $\alpha$ Are Key Mediators of Moraxella catarrhalis Triggered Exacerbation of Allergic Airway Inflammation.

Front. Immunol. 8:1562. doi: 10.3389/fimmu.2017.01562
Alterations of the airway microbiome are often associated with pulmonary diseases. For example, detection of the bacterial pathogen Moraxella catarrhalis in the upper airways is linked with an increased risk to develop or exacerbate asthma. However, the mechanisms by which $M$. catarrhalis augments allergic airway inflammation (AAl) remain unclear. We here characterized the cellular and soluble mediators of $M$. catarrhalis triggered excacerbation of $\mathrm{AAl}$ in wt and IL-17 deficient as well as in animals treated with TNF- $\alpha$ and IL-6 neutralizing antibodies. We compared the type of inflammatory response in $M$. catarrhalis infected, house dust mite (HDM)-allergic and animals infected with $M$. catarrhalis at different time points of HDM sensitization. We found that airway infection of mice with $M$. catarrhalis triggers a strong inflammatory response with massive neutrophilic infiltrates, high amounts of IL- 6 and TNF- $\alpha$ and moderate levels of CD4 ${ }^{+}$ T-cell-derived IFN- $\gamma$ and IL-17. If bacterial infection occurred during HDM allergen sensitization, the allergic airway response was exacerbated, particularly by the expansion of Th17 cells and increased TNF- $\alpha$ levels. Neutralization of IL-17 or TNF- $\alpha$ but not IL-6 resulted in accelerated clearance of $M$. catarrhalis and effectively prevented infectioninduced exacerbation of AAl. Taken together, our data demonstrate an essential role for TNF- $\alpha$ and IL-17 in infection-triggered exacerbation of AAl.

Keywords: exacerbation of pulmonary inflammation, IL-17, TNF- $\alpha$, Moraxellaceae infections, infection and allergy, exacerbation of allergic reactions, pulmonary inflammation, microbial exacerbation of pulmonary inflammation

\section{INTRODUCTION}

Constant exposure of the respiratory mucosa to pollutants, allergens, and pathogens requires robust and at the same time strictly regulated immune responses of the airways. A breakdown of controlled airway immune responses to these environmental factors may result in acute or chronic inflammation, including asthma, and chronic obstructive pulmonary disease (COPD). Respiratory tract infections have emerged as the most frequent triggers for pulmonary inflammation in both children and adults (1). Pathogenic bacteria including Streptococcus pneumoniae, Hemophilus influenzae, and Moraxella catarrhalis are microbial colonizers of the airway mucosa inducing an inflammatory immune response that prepares the ground for increased asthma susceptibility or exacerbation of established disease 
(2). Infection-triggered airway inflammation is often associated with increased amounts of inflammatory cytokines such as IL-6, IL-17, and TNF- $\alpha$ (3) and infiltrates of neutrophils, eosinophils, and different subtypes of T helper cells $(4,5)$ which seem to be related to the severity and pathogenesis of pulmonary inflammation (6). Paradoxically, many of these cytokines which induce pulmonary inflammation are also involved in antimicrobial host defense $(7,8)$, e.g., IL-17 and TNF- $\alpha$ mediate influx of neutrophils mediating first-line defense through uptake and killing of microbes. Although M. catarrhalis is a common pathogen known to trigger or exacerbate established pulmonary inflammation, the molecular and cellular mechanism still remains obscure (9).

$M$. catarrhalis is able to efficiently adhere to the epithelium of distinct mucosal tissues such as lung and nasopharynx. Together with S. pneumoniae and $H$. influenzae, $M$. catarrhalis is one of the major pathogens causing otitis media (OM) in children. Furthermore, several studies have reported that $M$. catarrhalis contributes to the exacerbations of COPD in adults (10). During the course of infection, $M$. catarrhalis induces a robust inflammatory response characterized by infiltrations of macrophages, lymphocytes, and neutrophils into infected tissue, which probably causes the pathogenesis of $\mathrm{OM}$ and also leads to exacerbations of COPD. The investigation of the molecular interplay between $M$. catarrhalis and host immune system has revealed that the recognition of this pathogen by multiple toll-like receptors (TLRs) such as TLR4 and TLR9 triggers the production of pro-inflammatory cytokines IL- 6 and TNF- $\alpha$ (11). In many asthma patients, $M$. catarrhalis is one of the dominant pathogen species found within the airway bacterial community (12). Although a link between infection with $M$. catarrhalis and exacerbation of asthma has been proposed, no functional data exist to our knowledge, indicating that this pathogen is causative for exacerbation of this disease.

Our aim was to investigate whether $M$. catarrhalis infection has the potential to exacerbate allergic airway inflammation (AAI) and if yes, to analyze the underlying pathomechanisms. Therefore, we established for the first time a murine model of $M$. catarrhalis airway infection and rigorously analyzed the mechanisms of pulmonary inflammation triggered exclusively by the pathogen or during developing or established AAI against the house dust mite (HDM) allergen.

We here show that airway infection with $M$. catarrhalis augments the phenotype of AAI mainly via TNF- $\alpha$ and T-cell derived IL-17 but not IL- 6 . Yet, in the absence of IL-17 and/or TNF- $\alpha$, infection-triggered lung inflammation as well as exacerbation of AAI was very mild and resulted in accelerated clearance of pathogenic airway bacteria, emphasizing the inflammation promoting role of these cytokines.

\section{RESULTS}

\section{Characteristics of Airway Inflammation Caused by $M$. catarrhalis infection}

Before investigating the consequences of bacterial airway infection on AAI, we established a murine infection model to first study the immune responses triggered by $M$. catarrhalis. Two hours after intranasal inoculation, infection was fully established and bacterial titers of the lung dropped massively at day 2 p.i. and were below detection at day 6 , revealing clearance of M. catarrhalis in normal animals (Figure 1A). It has to be noted that approx. $50 \%$ of wt animals intranasally infected with $M$. catarrhalis showed moribund symptoms at day 3 p.i. (Figure 2G). Total cell numbers in bronchoaleveolar lavage (BAL) increased 1 day after intranasal inoculation, peaked at day 4, and then decreased. M. catarrhalis infection caused airway inflammation primarily through massive influx of neutrophils and lower numbers of eosinophils and macrophages reaching their maximum at day 4 , followed by an increase in lymphocytes which peaked at day 7 p.i. Twelve days after infection, all BAL cells numbers returned to baseline level (Figures 1B,C). Accordingly, early and strong expression of CXCL1 and CXCL10 in the BAL was observed, both known to attract neutrophils and other inflammatory cells (Figure 1D). As M. catarrhalis infection causes airway inflammation $(9,13)$, we performed a kinetic study of inflammatory cytokines in the BAL. While IL-6, TNF- $\alpha$ and IL- $1 \beta$ peaked at day 1 p.i. and rapidly decreased to low levels by day 4 , the amounts of IFN- $\gamma$ and IL-17 were highest at day 7 p.i., coinciding with the peak of BAL lymphocytes (Figure 1E). Due to the late occurrence of IL-17 in the BAL, we continued to analyze the mRNA expression of this cytokine in lung homogenates. In accordance with BAL data, strong IL-17 mRNA expression was found at day 7 , correlating with the rise of $\mathrm{IL}-17^{+} \mathrm{CD} 4^{+} \mathrm{T}$ cells at this time point (Figures 1F,G). To identify the type of $\mathrm{T}$ cells producing pulmonary IL-17 after $M$. catarrhalis infection, lung lymphocytes were analyzed by flow cytometry. The majority of IL-17 secreting cells in the lung were conventional CD4 $4^{+} \mathrm{T}$ cells (70\%) which increased between days 7 and 15 p.i. and only a minor fraction of TCR $\gamma^{+} \delta^{+}$cells (14\%) was positive for IL-17 (Figure 1H). We next investigated inflammatory responses in IL-17 deficient mice. Interestingly, despite similar initial bacterial colonization of the lungs, IL-17 KO mice showed increased bacterial clearance as compared to wt animals (Figure 2A). Furthermore, these mice revealed significantly reduced total and differential BAL cell counts (Figures 2B,C) and lung tissues showed a massive reduction of cellular infiltrates as compared to wt animals (Figure 2D). The amounts of IL-6 and TNF- $\alpha$ in BAL and serum of infected IL-17 KO were diminished and mice did not succumb to $M$. catarrhalis infection wt animals (Figures 2E-G). Interestingly, despite the lack of IL-17, the number of neutrophils in the lung at day 1 p.i. was comparable to wt animals. Similar amounts of the CXCL1, 5, and 10 chemokines in IL-17 KO mice might compensate for early neutrophil attraction, independently in IL-17 (Figure S1 in Supplementary Material). Likewise, neutralization of TNF- $\alpha$, which is also produced by epithelial cells upon M. catarrhalis infection (Figure S2 in Supplementary Material), resulted in reduction of local and systemic IL-1 $\beta$, IL-6, and IL-17 and subsequent protection against $M$. catarrhalis infection (Figures 3A-C). Interestingly, in contrast to IL-17 and TNF- $\alpha$, we found that neutralization of IL- 6 increased local and systemic TNF- $\alpha$ as well as early IL- $1 \beta$ and IL-17 levels in the BAL. Anti-IL-6 treatment resulted in slightly enhanced numbers of animals with moribund symptoms, suggesting that IL-6 exerts an anti-inflammatory function during pulmonary $M$. catarrhalis infection (Figures 4A-C). 


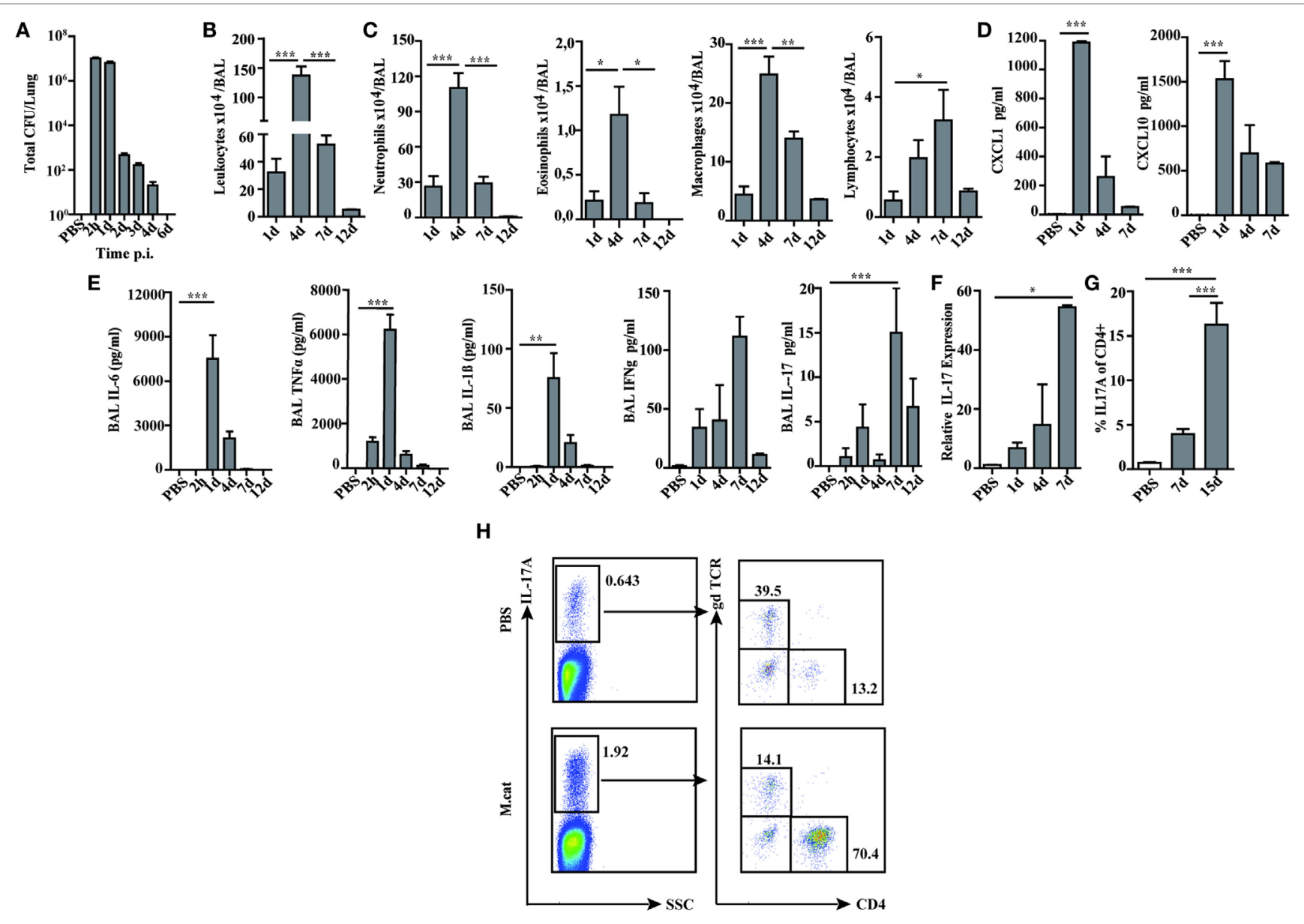

FIGURE 1 | Pulmonary immune response after intranasal $M$. catarrhalis infection. C57BI/6 animals were infected (i.n.) with $2 \times 10^{8} \mathrm{CFU} \mathrm{M}$. catarrhalis and BAL and lungs were harvested at indicated time points. (A) Bacterial CFU were determined in lung homogenates. (B) Total cell counts in BALF. (C) Differential cell counts in BALF. (D) CXCL1 and CXCL10 chemokines in BALF. (E) Cytokines in BALF. (F) Quantitative expression of IL-17 mRNA (qPCR) in lungs. (G) Frequency of $\mathrm{IL}-17 \mathrm{~A}^{+} \mathrm{CD} 4^{+} \mathrm{T}$ cells in lungs. $\mathbf{( H )}$ Analysis of $\gamma \delta \mathrm{T}$ cells and $\mathrm{CD} 4^{+} \mathrm{T}$ cells secreting IL-17A at day 7 after PBS treatment or infection. $n=6$ mice per group and two independent experiments were performed. ${ }^{\star \star \star} P=0.001,{ }^{\star \star} P=0.01$, and ${ }^{\star} P=0.05$ (one-way ANOVA). BAL, bronchoaleveolar lavage; BALF, BAL fluid; qPCR, quantitative polymerase chain reaction; PBS, phosphate-buffered saline; ANOVA, analysis of variance.

\section{Pulmonary Infection with Moraxella catarrhalis Exacerbates HDM-Induced AAI}

To study the mechanisms of $M$. catarrhalis caused exacerbation of AAI, wt C57Bl/6 mice (white bars) were intranasally infected with $M$. catarrhalis after the second exposure with HDM and analyzed on day 23 as shown in Figure 5A. While HDM exposure induced airway inflammation, additional infection of HDM sensitized wt mice with $M$. catarrhalis led to hugely increased numbers of leukocytes in the BALF mainly through infiltrating neutrophils, eosinophils, and lymphocytes (Figures 5B,C). This was also evident in lung histology, showing increased airway inflammation, goblet cell hyperplasia, and mucus production in M. catarrhalis infected, HDM-allergic mice (Figures 5D-F). To further study the cytokine pattern of pulmonary CD4+ $4^{+}$-cell populations, we compared HDM allergic mice with those that additionally contracted an infection. The latter group revealed significantly increased frequencies of IFN- $\gamma$, IL-17A, and IL- $5^{+} /$ $\mathrm{IL}_{-13^{+}}$double positive $\mathrm{CD} 4^{+} \mathrm{T}$ cells, although the absolute frequencies of IL- $5^{+} / \mathrm{IL}-13^{+}$Th2 cells were low as compared to those of IFN- $\gamma^{+}$and IL- $17^{+} \mathrm{CD} 4^{+} \mathrm{T}$ cells (Figure 5G; Figure S5A in Supplementary Material). Accordingly, increased amounts of IL-17 and IFN- $\gamma$ were found in the lungs of HDM allergic wt mice (white bars) concomitantly infected with $M$. catarrhalis (Figure S5B in Supplementary Material). Of note, cellular infiltrates and cytokine responses measured at day 23 were significantly lower than during acute infection with $M$. catarrhalis.

We then wondered whether the time of $M$. catarrhalis infection (prior to, during or after complete HDM sensitization) has an influence on exacerbation of AAI. Similar to infection during HDM sensitization, bacterial infection after established allergic reactions increased total BAL leukocyte counts mainly by neutrophils and lymphocytes. Together with increased lymphocytic infiltration, airway infection enhanced the frequencies of lung IL-17, IFN- $\gamma$, and IL-5 secreting CD4 ${ }^{+}$T cells as compared to with the HDM-only group (Figure S3 in Supplementary Material). Furthermore, we wondered whether a previously resolved 


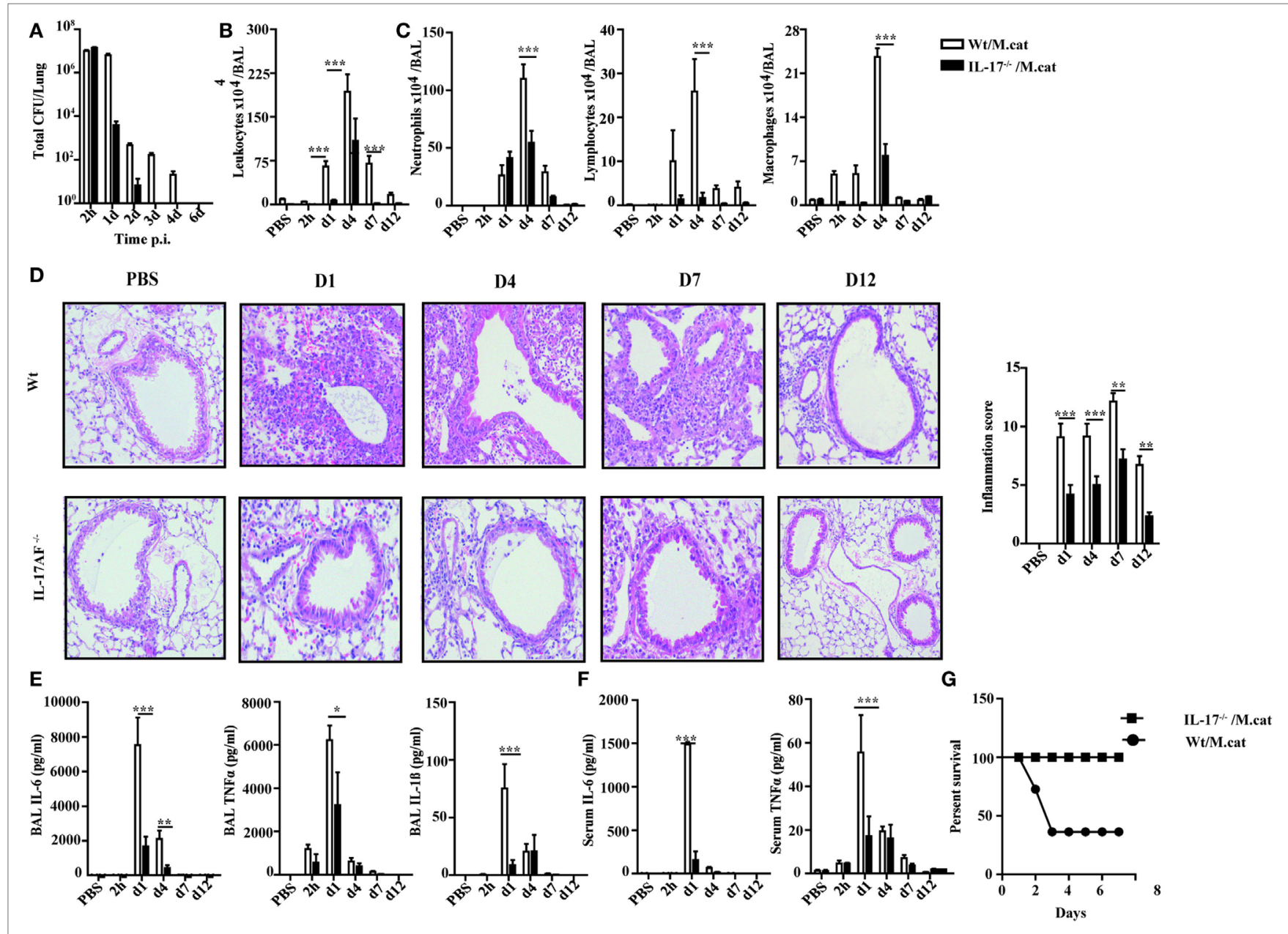

FIGURE 2 | Reduced inflammatory response to M. catarrhalis infection in IL-17 KO mice. IL-17 KO (black bars) and wt mice (white bars) were infected (i.n.) with $2 \times 10^{8}$ CFU M. catarrhalis. BAL, lungs, and serum were harvested at indicated time points. (A) Bacterial CFU in lung homogenates were counted at different time points after infection. (B) Airway inflammation represented by BAL total cell counts. (C) Differential cell counts in BAL. (D) Representative periodic acid Schiff-stained airways of wt and IL-17 KO mice (E) Amounts of IL-6, TNF- $\alpha$, and IL-1 $\beta$ in BALF. (F) IL-6 and TNF- $\alpha$ in serum. (G) Survival of IL-17 KO (squares) and wt animals (circles) after infection. Data were from two independent experiments $\left(n=10\right.$ mice per group). ${ }^{\star \star \star} P=0.001,{ }^{\star \star} P=0.01$, and ${ }^{\star} P=0.05$ (one-way ANOVA). BAL, bronchoaleveolar lavage; BALF, BAL fluid; ANOVA, analysis of variance.

infection with $M$. catarrhalis is still able to exacerbate HDM allergic reactions. Similar to infection during or after HDM sensitization, infection prior to allergen exposure triggered airway infiltrations of $\mathrm{CD}^{+} \mathrm{T}$ cells secreting increased amounts of IL-17, IFN- $\gamma$, and IL-5 (Figure S4 in Supplementary Material).

\section{IL-17 Is a Central Mediator of Infection-Triggered Exacerbation of AAI}

Since IL-17 has been reported to be associated with mucoepithelial infections and allergic responses (14), we next tested IL-17 deficient mice for the development of HDM-allergic airway responses. Interestingly, wt animals (white bars) and IL-17 KO mice (black bars) revealed similar numbers of neutrophils, eosinophils, and lymphocytes in the BALF (Figures 5B,C). Lung histology revealed slightly reduced pulmonary infiltrates in IL-17 KO mice as compared to with wt mice after HDM exposure (Figure 5D). Accordingly, airway inflammation, goblet cell hyperplasia, and mucus production were marginally diminished in IL-17 KO mice as compared to with controls (Figures 5E,F).

As early antibacterial immunity is mediated by IL-17controlled influx of neutrophils into infected tissues (15), we next investigated the role of this cytokine to trigger infection-induced exacerbation of AAI. IL-17 KO and wt mice were infected during the second HDM allergen exposure and analyzed at day 23 , as shown in Figure 5A. The data demonstrate that in contrast to wt animals, the number of neutrophils, eosinophils, and lymphocytes in the BALF of infected IL-17 KO mice was comparable to the non-infected, HDM group. Furthermore, comparable numbers of mucus secreting cells and similar inflammation scores suggest IL-17 as key cytokine of $M$. catarrhalis-induced exacerbation of AAI (Figures 5D-F). Yet, $M$. catarrhalis infection of HDM allergic IL-17 KO mice still enhanced the frequency of pulmonary IFN- $\gamma$ and IL-5/IL- 13 secreting CD $4^{+} \mathrm{T}$ cells (Figure 5G; Figures S5A,B in Supplementary Material). 

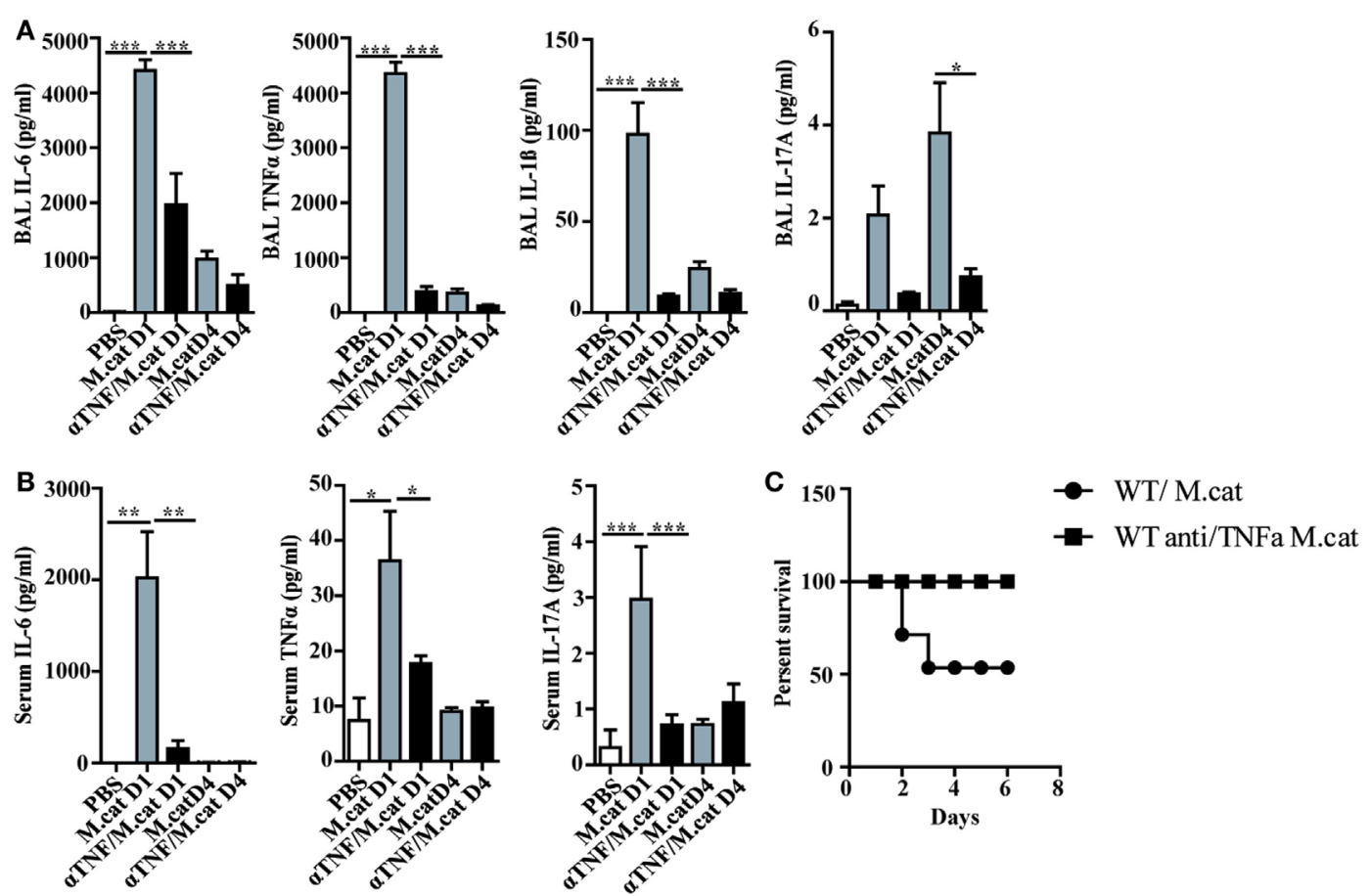

FIGURE 3 Neutralization of TNF- $\alpha$ protects against respiratory $M$. catarrhalis infection. C57BL/6 animals received a single i.p. injection of $100 \mu \mathrm{g}$ of anti-TNF- $\alpha$ (black bars) or IgG1 control mAbs (gray bars) and were $4 \mathrm{~h}$ later intranasally infected with $2 \times 10^{8} \mathrm{CFU}$ M. catarrhalis. (A) Amounts of IL-6, TNF- $\alpha$, IL-1 $\beta$, and IL-17 in BALF of untreated or anti-TNF- $\alpha$-treated mice at indicated time points. (B) As in (A) but in serum (C) Survival of anti-TNF- $\alpha$-treated animals (circle) or control lgG-treated (square) mice after infection with $M$. catarrhalis. Data were from two independent experiments, $n=6$ mice per group. ${ }^{* \star *} P=0.001,{ }^{\star *} P=0.01$, and ${ }^{*} P=0.05$ (one-way ANOVA). BALF, BAL fluid; ANOVA, analysis of variance.

Taken together, these findings show that IL-17 essentially contributes to $M$. catarrhalis triggered exacerbation of AAI.

\section{Anti TNF- $\alpha$ Treatment Attenuating IL-17-Dependent Exacerbation of Infection-Induced AAI}

It has been shown that neutrophil recruitment during inflammation is mediated by synergistic effects of IL- 17 and TNF- $\alpha$ in endothelial cells by enhancing the expression of P- and E-selectin (16). We thus wondered whether neutralization of TNF- $\alpha$ also attenuates infection-induced exacerbation of AAI. Briefly, HDM sensitized mice were treated only once with anti-TNF- $\alpha \mathrm{mAb} 4 \mathrm{~h}$ before intranasal infection with $M$. catarrhalis and analyzed at day 23, as depicted in Figure 6A. Control groups included mice that received phosphate-buffered saline (PBS) or HDM alone as well as infected, HDM sensitized animals.

Differential cell counts of BAL cells revealed that predominantly neutrophils were reduced by anti-TNF- $\alpha$ treatment, while the numbers of macrophages were increased when compared with $\mathrm{HDM} /$ infected controls. The increase of BAL macrophages after TNF- $\alpha$ neutralization seems to compensate for the low number of neutrophils in order to eliminate M. catarrhalis. Interestingly, the number of eosinophils or lymphocytes were not significantly reduced after TNF- $\alpha$ treatment as shown in Figures 6B,C. Lung histology confirmed that anti-TNF- $\alpha$ treatment vastly reduced infection-triggered inflammatory infiltrates in HDM allergic animals which was also reflected by the decreased number of $\mathrm{PAS}^{+}$ cells and the low-inflammation score (Figures 6D-F). We next wondered whether TNF- $\alpha$ neutralization also affects pulmonary T-cell activation. Anti-TNF- $\alpha$ treatment attenuated the activation of IFN- $\gamma^{+}$and IL- $17^{+}$lymphocytes but had no influence on the low frequency of IL-5/IL-13 secreting, pulmonary $\mathrm{CD} 4^{+} \mathrm{T}$ cells (Figure 6G; Figure S6 in Supplementary Material).

In summary, the experiments revealed that a single application of anti-TNF- $\alpha \mathrm{mAb}$ is sufficient to markedly attenuate M. catarrhalis-induced exacerbation of AAI.

\section{DISCUSSION}

We here show that infection with $M$. catarrhalis exacerbates HDM-triggered AAI mainly by an IL-17- and TNF- $\alpha$-dependent inflammatory response. Lack of IL-17 or neutralization of TNF- $\alpha$ prevented infection-triggered exacerbation of allergic pulmonary disease.

Infection with $M$. catarrhalis has been shown to be an important risk factor for newborns or patients with chronic pulmonary diseases to either develop asthma or to exacerbate disease symptoms (17). While evidence suggests that the frequency of respiratory infections rather than the type of pathogen influences asthma development (15), the immunological mechanisms of infectiontriggered exacerbation of AAI are not entirely understood, which is reflected by the limited therapeutic options. 

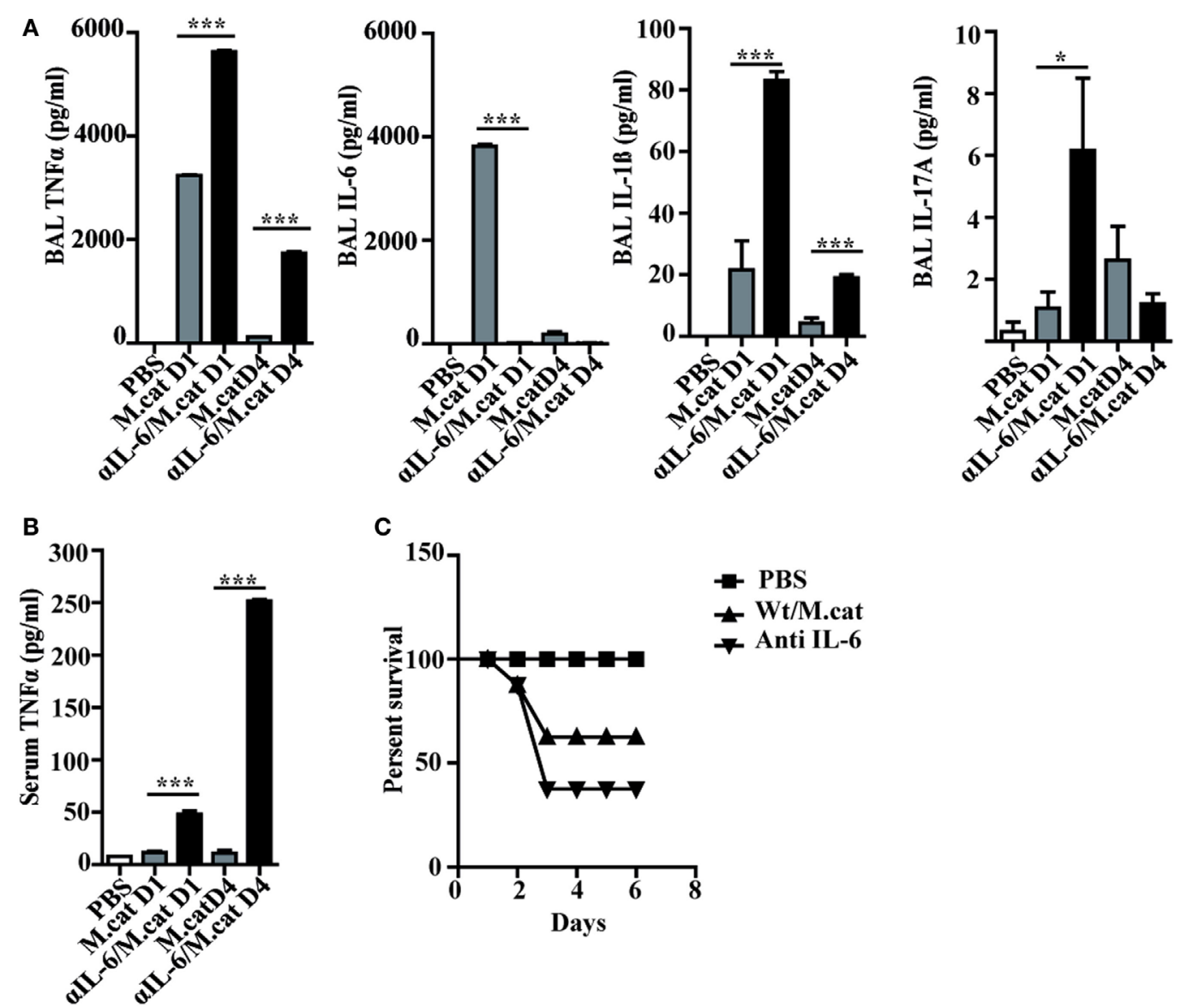

FIGURE 4 | Neutralization of IL-6 enhances M. catarrhalis triggered inflammation. C57BL/6 animals received a single i.p. injection of 100 Hg of anti-IL-6 or IgG1 control mAbs and $4 \mathrm{~h}$ later were infected (i.n.) with $M$. catarrhalis ( $2 \times 10^{8}$ CFU) (A) Amounts of TNF- $\alpha$, IL-6, IL-1 $\beta$, and IL-17 in BALF. (B) Amount of TNF- $\alpha$ in serum. (C) Survival of PBS-treated control mice (PBS), control-lgG1-treated animals infected with M.catarrahlis (Wt/M.cat) and anti-IL-6-mAb-treated animals infected with M. catarrhalis (anti IL-6). Data were from two independent experiments, $n=3$ mice per group. ${ }^{\star \star \star} P=0.001$, ${ }^{\star \star} P=0.01$, and ${ }^{\star} P=0.05$ (one-way ANOVA). BALF, BAL fluid; PBS, phosphate-buffered saline; ANOVA, analysis of variance.

Infection with $M$. catarrhalis during HDM allergen exposure strongly increased the influx of neutrophils, eosinophils, and $\mathrm{T}$ cells, while infection after established allergic inflammation caused exacerbation primarily by neutrophils. In contrast, infection prior to HDM exposure predominantly triggered the expansion of pulmonary T-lymphocyte populations. However, common to all three settings of infection is the induction of a mixed pulmonary Th1, Th2, and Th17 immune response. Thus, our data suggest that bacterial infection influences the development and outcome of AAI irrespective of the time-point of allergen exposure. This reflects the situation in patients where viral and/or bacterial infections in early life or during asthma have been associated with the development or exacerbation of the disease, respectively (18).

The importance of IL-17 in protection against pulmonary bacterial infection was shown for several pathogens, including Klebsiella pneumoniae, Mycoplasma pneumoniae, Bordatella pertussis, and Mycobacterium tuberculosis (19). Accordingly, IL-17 receptor KO mice revealed increased susceptibility to bacterial infections due to impaired neutrophil and macrophage recruitment to the site of bacterial entry (20). On the other hand, the expression of IL-17 in the airways of asthma patients was shown to correlate with neutrophilic lung inflammation (21-23). Despite this ambiguous role, our data revealed that IL-17 deficient animals cleared the pathogen even faster than control littermates. Unimpaired expression of CXCL1, 5, and 10 chemokines in IL-17 $\mathrm{KO}$ mice seems to compensate for early neutrophil influx but not for later stages of infection where the pulmonary neutrophil response was decreased in $\mathrm{KO}$ animals.

Infection of human respiratory tract epithelial cells with $M$. catarrhalis has been shown to increase various pro-inflammatory genes, e.g., TNF- $\alpha$, IL- $1 \beta$, and IL-17 (10). We observed a similar cytokine response in $M$. catarrhalis infected wt but not IL-17 KO mice which showed even increased resistance against infection. Furthermore, in the absence of IL-17, the mixed neutrophilic/eosinophilic airway inflammation of HDM challenged wt mice switched to an attenuated, almost exclusive eosinophilic response. Likewise, $M$. catarrhalis infection of HDM allergic mice did not lead to exacerbation of pulmonary inflammation in IL-17 deficient mice. Taken together, these findings indicate 
A

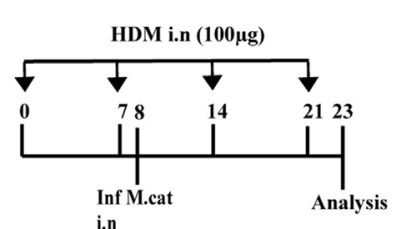

i.n

D
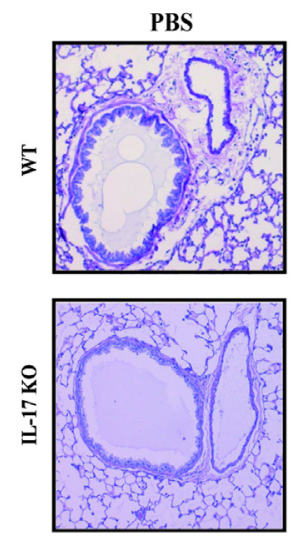

HDM
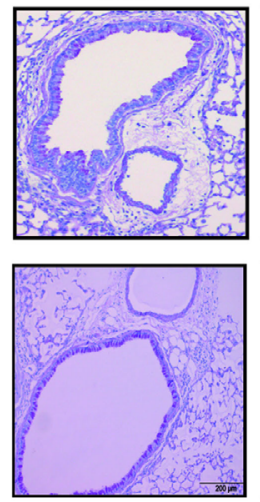
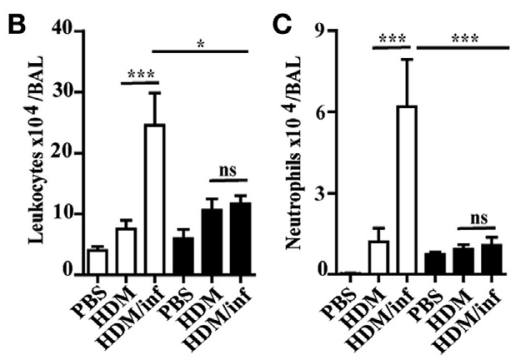
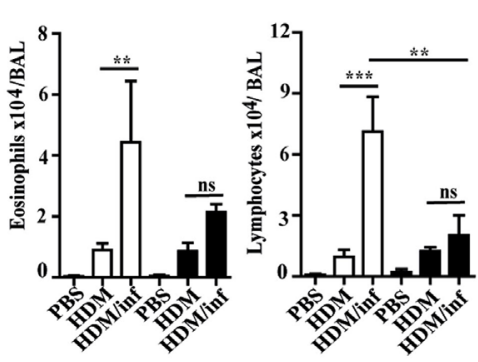

चIt

HDM/inf

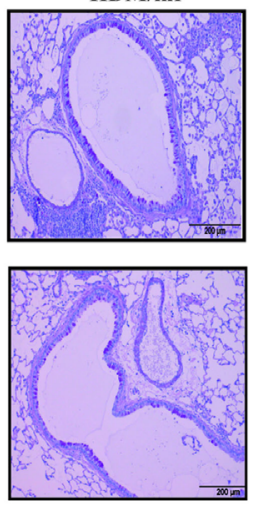

$\mathbf{E}$

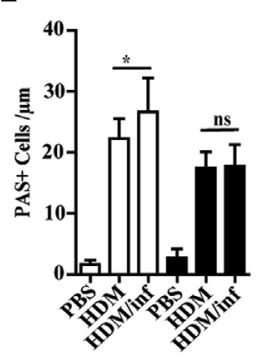

$\mathbf{F}$
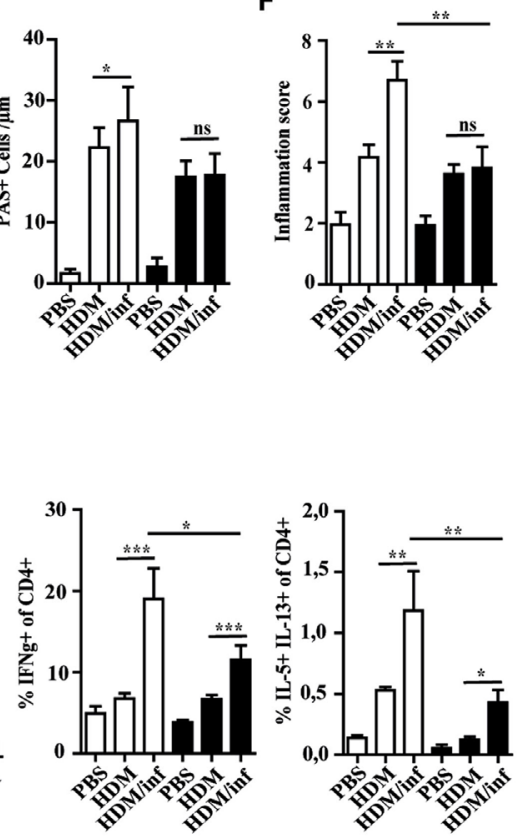

FIGURE 5 | IL-17 is a key cytokine of M. catarrhalis-induced exacerbation of AAl. C57BL/6 (white bars) and IL-17 KO animals (black bars) were infected (i.n.) with $2 \times 10^{8} \mathrm{CFU}$ M. catarrhalis after the second HDM exposure. BAL and lungs were analyzed at day 23. (A) Protocol. (B) Total cell counts in BAL. (C) Differential cell counts. (D) Representative periodic acid Schiff-stained airways. (E) Goblet cell counts in lung tissues. (F) Inflammation score. (G) Intracellular staining of CD4+ lung T cells for IL-17A, IFN- $\gamma$, IL-5, and IL-13. Data were from two independent experiments, $n=8$ mice per group. ${ }^{\star \star \star} P=0.001,{ }^{\star \star} P=0.01$, and ${ }^{\star} P=0.05$ (one-way ANOVA). AAl, allergic airway inflammation; HDM, house dust mite; BAL, bronchoaleveolar lavage; ANOVA, analysis of variance.

that pulmonary infection is a crucial trigger for IL-17-induced, exacerbation of neutrophilic inflammation.

Besides IL-17, IL-6 has been considered as promising target to reduce pathogenesis of asthma and COPD, particularly those of eosinophilic/neutrophilic phenotype $(24,25)$. IL-6 is known to mediate pro-inflammatory and anti-inflammatory immune functions via trans- and classical signaling, respectively (26). As anti-IL-6 treatment led to enhanced secretion of local and systemic TNF- $\alpha$ with high susceptibility to $M$. catarrhalis infection, we did not consider IL-6 as appropriate target for treatment.

The contradicting reports about efficiency and safety of TNF- $\alpha$ blockers in animal models and patients with severe asthma (27, 28) prompted us to study the effect of TNF- $\alpha$ neutralization during pulmonary infection and exacerbation of AAI.

We found that neutralization of TNF- $\alpha$ prior to pulmonary M. catarrhalis infection resulted in a substantial decline of IL-6,
Il-1 $\beta$, and IL-17, together with increased survival of infected animals. Furthermore, TNF- $\alpha$ neutralization was able to effectively reduce infection-induced exacerbation of HDM-allergic reactions despite minor or no reduction of pulmonary Th1, Th17, and Th2 cells, respectively. Even though we found a strong decrease of neutrophils in the BAL, the mechanisms by which TNF- $\alpha$ acts in asthmatic airways might be manifold: Besides its effect to recruit neutrophils and eosinophils (29), it has been shown to enhance T-cell responses (30), to promote glucocorticoid resistance and airway remodeling (31).

While it has been shown that TNF- $\alpha$ or IL-17 alone are able to induce inflammatory responses, inflammation was enhanced in the presence of both cytokines $(32,33)$.

In line with these observations, our data demonstrate that neutralization of IL-17 or TNF- $\alpha$ is similar effective in attenuating exacerbation of AAI. Whether neutralization of these cytokines is also suitable for therapy still remains to be determined. Since 


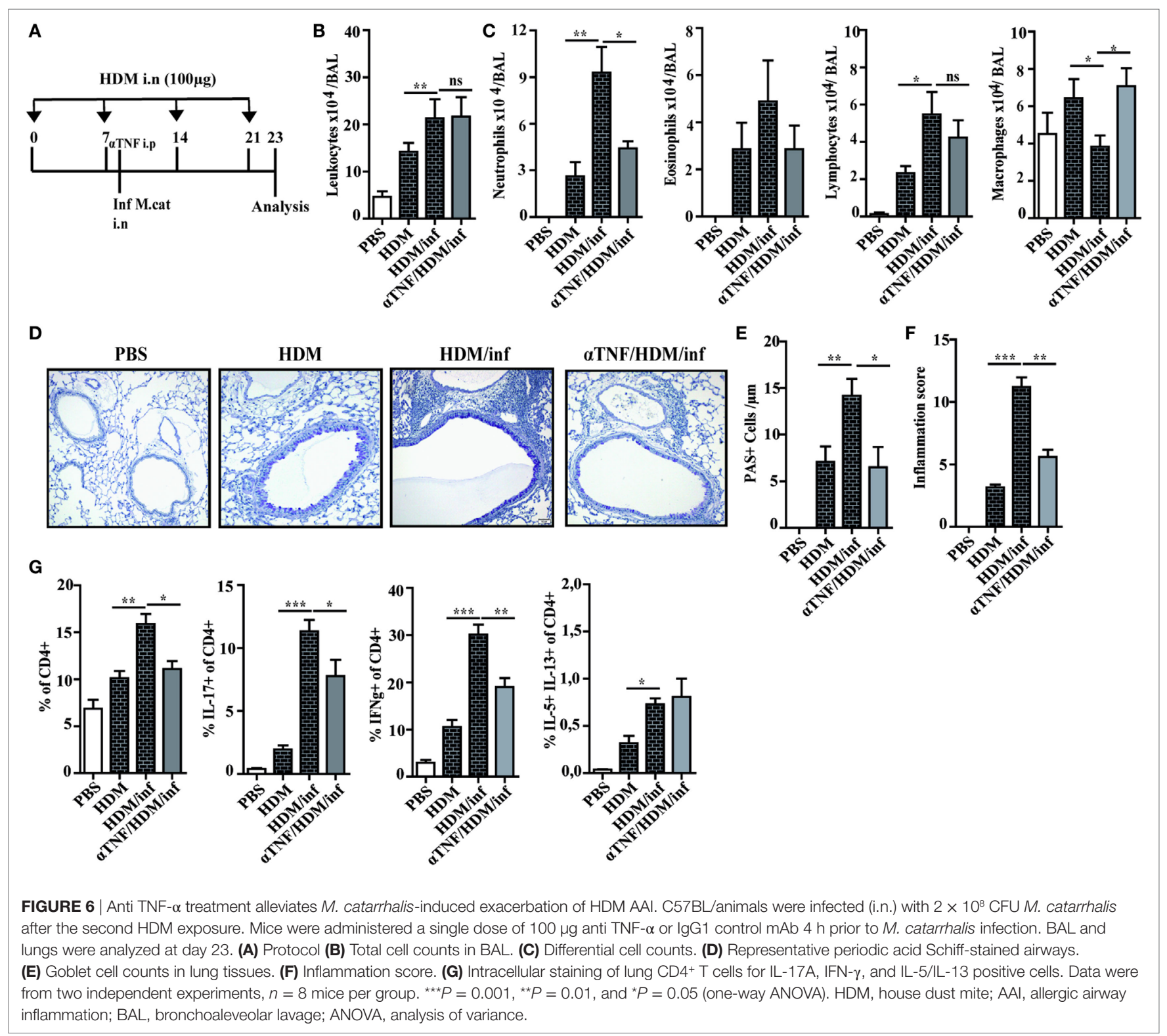

prolonged anti TNF- $\alpha$ or IL-17 therapies bear the risk of severe infections or development of metaplastic malignancies (28, $34,35)$, only short-term treatment with combined anti-IL-17/ anti-TNF- $\alpha$ antibodies might be qualified for the treatment of infection-triggered exacerbation of AAI.

\section{MATERIALS AND METHODS}

\section{Animals}

Female 8-10-weeks-old C57BL/6J mice were purchased from Charles River Laboratories, Germany. Female IL-17AF ${ }^{-1-}$ mice on C57BL/6J background were kindly provided by Prof. Dr. Immo Prinz (Hannover, Germany) and housed in a pathogenfree facility with individually ventilated cages. All experiments were approved by the Regierunspräsidium Gießen (Protocol no. MR 20/6 Nr. 123/2012).

\section{Bacteria and Infection}

A patient isolate of $M$. catarrhalis was stored at $-80^{\circ} \mathrm{C}$ in glycerol. Bacteria from frozen stocks were grown aerobically in Columbia blood agar plates (BBL; Becton Dickinson, MD, USA) with $5 \%$ sheep blood at $37^{\circ} \mathrm{C}$ overnight, washed off the plate and resuspended in sterile PBS for infection. Anesthetized mice were inoculated intranasally (i.n.) with $2 \times 10^{8} \mathrm{CFU}$ $M$. catarrhalis resuspended in $50-\mu \mathrm{L}$ PBS, before during or after HDM challenge.

\section{Determination of CFU in Organs}

Lungs were aseptically removed and homogenized in $1 \mathrm{~mL}$ of sterile PBS.

Serial dilutions of BAL fluid and lung homogenates were prepared in sterile PBS, plated on Columbia blood agar plates (BBL; Becton Dickinson, MD USA) with 5\% sheep blood and 
incubated overnight at $37^{\circ} \mathrm{C}$. Colonies were enumerated and bacterial numbers per lung calculated.

\section{Experimental Model of Acute HDM AAI}

8-10-week-old C57Bl/6 mice were slightly anesthetized (Ketamin/2\%Rompun, i.p.) and weekly challenged by intranasal (i.n.) administration of 100- $\mu$ g HDM extract (GREER, Lenoir, USA) in $50-\mu \mathrm{L}$ PBS on days $0,7,14$, and 21 . Two days after last HDM challenges mice were sacrificed and blood, bronchoalveolar lavage (BAL) fluids, lungs were collected for further analysis.

\section{Administration of Neutralizing Antibodies}

To assess the role of IL- 6 and TNF- $\alpha$ during the infection with M. catarrhalis, neutralizing anti-IL-6 (clone MP5-20F3) or antiTNF- $\alpha$ (clone MP6-XT22) antibodies were used. Rat IgG1 isotype control was applied for treatment of control mice. Four hours before M. catarrhalis infection, mice were treated intraperitoneally once with $100 \mu \mathrm{g}$ of neutralizing antibodies, respectively. All used antibodies were generated and purified by Manuela Staeber (Max Planck Institute for Infection Biology, Berlin, Germany).

\section{Bronchoaleveolar Lavage}

Bronchoaleveolar lavage was performed $48 \mathrm{~h}$ after the last challenge using 1-mL cold PBS supplemented with a protease inhibitor cocktail (Roche, Mannheim, Germany). An automated Casy TT cell counter (Schaerfe System, Reutlingen, Germany) was used to determine the total leukocyte cell counts. Cells were centrifuged and the cell-free supernatant was stored at $-20^{\circ} \mathrm{C}$ until cytokines were measured by Elisa. For differential cell counts, Cytospin preparations were fixed and then stained with DiffQuick (Merz \&Dade AG, Dudingen, Switzerland). Macrophages, lymphocytes, eosinophils, and neutrophils were identified by standard morphologic criteria and 300 cells were counted per cytospin. The measurement of cytokines IFN- $\gamma$, IL-17, and chemokines (CXCL1, CXCL5, and CXCL10) was performed by Cytometric Bead Array (BD, Heidelberg, Germany) according to manufacturer's protocol.

\section{Histology of the Airways}

Directly after BAL, lungs were fixed with $10 \%$ formalin via the trachea, removed, and stored in $10 \%$ formalin. Lung tissues were embedded into paraffin. Tissues were cut in $3-\mu \mathrm{m}$ sections and stained using hematoxylin and eosin (HE) as well as periodic acid Schiff (PAS). Goblet hyperplasia was assessed by cell counting and expressed as the number of goblet cells per $100-\mu \mathrm{m}$ basement membrane, according to Foster et al. (2000).

\section{Measurement of HDM-Specific Antibodies in Serum Samples}

The amount of HDM-specific IgG1 and IgG2c antibodies was measured by the ELISA. Maxi-Sorb plates were coated with $50-\mu \mathrm{g} /$ mL HDM extract in bicarbonate buffer ( $\mathrm{pH} 9.6)$ overnight at $4^{\circ} \mathrm{C}$. Subsequently, coated wells were blocked with $1 \%$ w/V BSA in PBS for $2 \mathrm{~h}$ at RT. After washing, serum samples $(1: 100)$ were incubated overnight at $4^{\circ} \mathrm{C}$, washed, and incubated with the corresponding biotin-labeled anti-immunoglobulin antibody overnight at $4^{\circ} \mathrm{C}$.
Plates were washed and incubated with streptavidin-peroxidase for $30 \mathrm{~min}$ at RT. After development with substrate, the reaction was stopped using $\mathrm{H} 2 \mathrm{SO} 4$ and ODs were read at $450 \mathrm{~nm}$.

\section{Measurement of Cytokines in BAL Fluid (BALF)}

TNF- $\alpha$, IL- 6 , IL-1 $\beta$, IL-5, IL-13, IFN- $\gamma$, and IL-17 were measured in cell-free lavage fluid by Cytometric Bead array (CBA; BD BIOsciences; San Diego, CA, USA) as described by the manufacturer.

\section{Quantitative Analysis of Cytokine Expression}

Expression of IFN- $\gamma$ and IL-17 mRNA was determined by quantitative real-time polymerase chain reaction (RT-PCR) analysis using LightCycler technology (Roche). RNA was extracted from lung tissues according to manufacturer's instructions. cDNA synthesis was performed after removal of contaminating genomic DNA by DNase treatment using Superscript reverse transcriptase (Invitrogen, Karlsruhe, Germany) as described by manufacturer. Quantitative PCR (QPCR) was performed by using the QuantiTect SYBR Green PCR kit (QIAgen) and primers specific for IFN- $\gamma$ (forward: 5' GCT TTG CAG CTC TTC CTC AT 3' and reverse 5' GCA GGA TTT TCA TGT CAC CA 3'), IL-17 (forward: 5' AAG GCA GCA GCA ATC ATC CC 3' and reverse 5' GGG TCT TCA TTG CGG TGG AG $3^{\prime}$ ) Results were expressed as $x$-fold expression in relation to the house keeping gene L-32.

\section{Flow Cytometry}

Lungs were cut into $2-5 \mathrm{~mm}$ pieces and incubated for $30 \mathrm{~min}$ at $37^{\circ} \mathrm{C}$ in the incubation medium (IM) (RPMI-1640 with $\mathrm{L}$ Glutamine and $\mathrm{NaHCO} 3,10 \%$ FCS, 16 non-essential amino acids (PAA, Pasching, Austria), $100 \mathrm{mg} / \mathrm{mL}$ streptomycin, $120 \mathrm{mg} /$ $\mathrm{mL}$ penicillin) supplemented with $1-\mathrm{mg} / \mathrm{mL}$ Collagenase $\mathrm{D}$ (Roche, Basel, Switzerland) and $20 \mu \mathrm{g} / \mathrm{mL}$ DNase I (Roche, Basel, Switzerland). The pre-digested lungs were minced through 100-mm nylon cell strainer (BD Falcon, NJ, USA) diluted with IM and centrifuged at $1,700 \mathrm{rpm}$ for $5 \mathrm{~min}$. Cell pellets were resuspended in erythrocytes lysis buffer $\left(8 \mathrm{~g} / \mathrm{L} \mathrm{NH} \mathrm{NH}_{4}\right.$; $1 \mathrm{~g} / \mathrm{L}$ $\mathrm{KHCO}_{3} ; 37.2 \mathrm{mg} / \mathrm{L}$ EDTA) and incubated at room temperature for 3-5 min. Cells were washed, centrifuged, and resuspended in IM supplemented with $50 \mathrm{ng} / \mathrm{mL}$ PMA, $750 \mathrm{ng} / \mathrm{mL}$ ionomycin, and $10 \mathrm{mg} / \mathrm{mL}$ brefeldin A for $4 \mathrm{~h}$. Extracellular staining was performed in PBS with 1\% FCS and presence Fc blocking antibody (clone 2.4G2, BD, Heidelberg, Germany) and fluorophorelabeled antibodies (eBioscience, San Diego, CA, USA, if not specified otherwise). The following antibodies for extracellular T-cell staining was performed: anti-CD4-V450 (RM4-5), antiCD8 $\alpha$-V500 (53-6.7), and anti-CD25-PE-Cy7 (PC61.5) (BD, Heidelberg, Germany). After extracellular staining, T cells were fixed with FoxP3-Fixation Kit (eBioscience, San Diego, CA, USA) and permeabilized with $0.3 \%$ Saponin, $1 \%$ FCS in PBS followed by an intracellular staining with the following fluorophore-labeled antibodies: IFN- $\gamma$-PerCP-Cy5.5 (XMG1.2, Biolegend, San Diego, CA, USA); IL-17-AlexaFluor 700 and FoxP3-AlexaFluor 700 (FJK-16 s); IL-5-PE (TRFK5); IL-13-AlexaFluor 647 [(eBio13A) (eBioscience, San Diego, CA, USA)]. Every staining included 
negative and isotype controls. Fluorescence signals were acquired by flow cytometry (FACSAria III; BD, Heidelberg, Germany) and analyzed using FACSDivaTM software.

\section{Statistical Analysis}

Data are expressed as \pm SEM. We performed one-way analysis of variance (ANOVA) statistical analysis followed by Bonferroni HSD test using GraphPad Prism 5 software. The values of $P<0.05$ were considered to be significant.

\section{ETHICS STATEMENT}

This manuscript does not include human studies.

\section{AUTHOR CONTRIBUTIONS}

US designed the study, evaluated the data, and wrote the manuscript. SA performed the experiments and evaluated data. $\mathrm{SH}$ contributed to the design of experiments. HR contributed to the design of experiments and evaluated Facs data. AK critically read the manuscript and evaluated data. RM organized and identified microbial material and discussed findings. IP contributed animals, evaluated data, and critically read the manuscript. OP performed experiments. AV evaluated data and critically read the manuscript. HG advised experiments, read the manuscript, and evaluated the data. AH contributed with experiments and GG advised experiments and evaluated Facs data.

\section{ACKNOWLEDGMENTS}

We thank many colleagues, particularly Thomas Ruppert, Claudia Trier, Helene Bykow for excellent technical assistance and advice. We thank the flow cytometry core facility Marburg for their support.

\section{FUNDING}

This work was supported by DAAD (to SA) and the DFG [(to US) (grant number STE 776/3-1)].

\section{SUPPLEMENTARY MATERIAL}

The Supplementary Material for this article can be found online at http://www.frontiersin.org/article/10.3389/fimmu.2017.01562/ full\#supplementary-material.

\section{REFERENCES}

1. Pelaia G, Vatrella A, Gallelli L, Renda T, Cazzola M, Maselli R, et al. Respiratory infections and asthma. Respir Med (2006) 100(5):775-84. doi:10.1016/j. rmed.2005.08.025

2. Larsen JM, Brix S, Thysen AH, Birch S, Rasmussen MA, Bisgaard H. Children with asthma by school age display aberrant immune responses to pathogenic airway bacteria as infants. J Allergy Clin Immunol (2014) 133(4):1008-13. doi:10.1016/j.jaci.2014.01.010

3. Lambrecht BN, Hammad H. The immunology of asthma. Nat Immunol (2015) 16(1):45-56. doi:10.1038/ni.3049
FIGURE S1 | Early neutrophil influx induced by Moraxella catarrhalis is independent of IL-17. WT and IL-17AF-/- mice were infected (i.n.) with $2 \times 10^{8} \mathrm{CFU} M$. catarrhalis. BALF and lungs were harvested at indicated time points. (A) Identification of BALF neutrophils by flow cytometry. Neutrophils were stained with Ly6C+ly6G+ cells on day one after infection. (B) Amounts of CXCL1, CXCL5, and CXCL10 chemokines in BALF, $n=5$ mice per group. Two independent experiments were performed ${ }^{\star \star \star} P=0.001,{ }^{\star \star} P=0.01$, and ${ }^{*} P=0.05$ (one-way ANOVA). BALF, BAL fluid; ANOVA, analysis of variance.

FIGURE S2 | Tracheal epithelial cells produce TNF-a upon Moraxella catarrhalis infection. Primary tracheal cells from C57BL/6 mice were cultured and left untreated or infected with indicated $\mathrm{MOI}$ of $\mathrm{M}$. catarrhalis. Culture supernatants were taken at indicated time-points and $\mathrm{MOI}$ and analyzed for secretion of TNF- $\alpha$. Data represent two independent experiments with triplicates for each treatment condition. ${ }^{\star \star \star} P=0.001,{ }^{\star \star} P=0.01$, and ${ }^{\star} P=0.05$ (one-way ANOVA). ANOVA, analysis of variance.

FIGURE S3 | Moraxella catarrhalis exacerbates established HDM-allergic inflammation. C57BL/6 animals were infected (i.n.) with $2 \times 10^{8} \mathrm{CFU}$ M. catarrhalis after the last HDM exposure at day 22. BAL and lungs were analyzed at day 29. (A) Protocol. (B) Total cell counts in BALF. (C) Differential cell counts. (D) Representative periodic acid Schiff-stained airways. (E) Goblet cell counts in lung tissues. (F) Inflammation score. (G) Intracellular staining of lung $\mathrm{CD}^{+} \mathrm{T}$ cells for IL-17A, IFN- $\gamma$, and IL-5. Data were from two independent experiments $\left(n=8\right.$ mice per group). ${ }^{* \star} P=0.001,{ }^{* *} P=0.01$, and ${ }^{*} P=0.05$ (one-one-way ANOVA). HDM, house dust mite; BAL, bronchoaleveolar lavage; BALF, BAL fluid; ANOVA, analysis of variance.

FIGURE S4 | Moraxella catarrhalis infection prior to HDM exposure enhances pulmonary T-cell responses. C57BL/6 animals were infected (i.n.) with $2 \times 10^{8} \mathrm{CFU} M$. catarrhalis. Seven days prior to HDM exposure. BAL and lungs were analyzed at day 23. (A) Protocol. (B) Total cell counts in BALF. (C) Differential cell counts. (D) Representative FACs analysis of pulmonary CD4+ T cells stained for IL-17A, IFN- $\gamma$ and IL-5/L-13. (E) Statistical distribution of T-cell populations (eight animals) corresponding to the analysis in $D$. Data were from two independent experiments, $n=4$ mice per group. ${ }^{\star \star *} P=0.001,{ }^{* \star} P=0.01$, and ${ }^{*} P=0.05$ (one-way ANOVA). HDM, house dust mite; BAL, bronchoaleveolar lavage; BALF, BAL fluid; ANOVA, analysis of variance.

FIGURE S5 | Analysis of cytokines in the lung of mice infected with Moraxella catarrhalis during HDM exposure. C57BL/6 and IL-17 KO animals were infected (i.n.) with $2 \times 10^{8} \mathrm{CFU} M$. catarrhalis after the second $\mathrm{HDM}$ exposure. Lungs were analyzed at day 23. (A) Intracellular staining of lung $C D 4^{+} T$ cells was performed for IL-17A, IFN- $\gamma$, IL-5, and IL-13. Dot plots are representative of three similar experiments. (B) Amounts of IFN- $\gamma, \mathrm{IL}-17, \mathrm{IL}-5$, and IL-13 were measured by ELISA in lung homogenates. Data were from three independent experiments ( $n=8$ mice per group). ${ }^{* *} P=0.01$ and ${ }^{*} P=0.05$ (one-way ANOVA). HDM, house dust mite; ANOVA, analysis of variance.

FIGURE S6 | Effects of anti-TNF- $\alpha$ on inflammatory cytokines production in M. catarrhalis infected, HDM allergic mice. C57BL/6 animals were infected (i.n.) with $M$. catarrhalis $\left(2 \times 10^{8} \mathrm{CFU}\right)$ after the second HDM exposure. Mice were treated i.p. with 100- $\mu \mathrm{g}$ anti-TNF- $\alpha$ or lgG1 control mAb, $4 \mathrm{~h}$ before infection with M. catarrhalis. Lung lymphocytes were analyzed at day 23. (A) Intracellular staining of lung CD4+ $T$ cells for IL-17A, IFN- $\gamma$, IL-5, and IL-13. Data were from two independent experiments $\left(n=16\right.$ mice per group). ${ }^{\star \star *} P=0.001,{ }^{* \star} P=0.01$, and ${ }^{*} P=0.05$ (one-way ANOVA). HDM, house dust mite; ANOVA, analysis of variance.

4. Vock C, Hauber HP, Wegmann M. The other T helper cells in asthma pathogenesis. J Allergy (2010) 2010:519298. doi:10.1155/2010/519298

5. Baraldo S, Oliani KL, Turato G, Zuin R, Saetta M. The role of lymphocytes in the pathogenesis of asthma and COPD. Curr Med Chem (2007) 14(21):2250-6. doi:10.2174/092986707781696573

6. Barnes PJ. The cytokine network in asthma and chronic obstructive pulmonary disease. J Clin Invest (2008) 118(11):3546-56. doi:10.1172/JCI36130

7. Adler KB, Fischer BM, Wright DT, Cohn LA, Becker S. Interactions between respiratory epithelial-cells and cytokines - relationships to lung inflammation. Ann N Y Acad Sci (1994) 725:128-45. doi:10.1111/j.1749-6632.1994. tb00275.x 
8. Brightling C, Berry M, Amrani Y. Targeting TNF-alpha: a novel therapeutic approach for asthma. J Allergy Clin Immunol (2008) 121(1):5-10; quiz 11-2. doi:10.1016/j.jaci.2007.10.028

9. N'Guessan PD, Haarmann H, Steiner T, Heyl K, Schreiber F, Heinrich A, et al. The Moraxella catarrhalis-induced pro-inflammatory immune response is enhanced by the activation of the epidermal growth factor receptor in human pulmonary epithelial cells. Biochem Biophys Res Commun (2014) 450(2):1038-44. doi:10.1016/j.bbrc.2014.06.102

10. de Vries SP, Eleveld MJ, Hermans PW, Bootsma HJ. Characterization of the molecular interplay between Moraxella catarrhalis and human respiratory tract epithelial cells. PLoS One (2013) 8(8):e72193. doi:10.1371/journal. pone. 0072193

11. Hassan F, Ren D, Zhang W, Merkel TJ, Gu XX. Moraxella catarrhalis activates murine macrophages through multiple toll like receptors and has reduced clearance in lungs from TLR4 mutant mice. PLoS One (2012) 7(5):e37610. doi:10.1371/journal.pone.0037610

12. Green BJ, Wiriyachaiporn S, Grainge C, Rogers GB, Kehagia V, Lau L, et al. Potentially pathogenic airway bacteria and neutrophilic inflammation in treatment resistant severe asthma. PLoS One (2014) 9(6):e100645. doi:10.1371/ journal.pone. 0100645

13. Parameswaran GI, Wrona CT, Murphy TF, Sethi S. Moraxella catarrhalis acquisition, airway inflammation and protease-antiprotease balance in chronic obstructive pulmonary disease. BMC Infect Dis (2009) 9:178. doi:10.1186/1471-2334-9-178

14. Ishigame H, Kakuta S, Nagai T, Kadoki M, Nambu A, Komiyama Y, et al. Differential roles of interleukin-17A and $-17 \mathrm{~F}$ in host defense against mucoepithelial bacterial infection and allergic responses. Immunity (2009) 30(1):108-19. doi:10.1016/j.immuni.2008.11.009

15. Ferretti S, Bonneau O, Dubois GR, Jones CE, Trifilieff A. IL-17, produced by lymphocytes and neutrophils, is necessary for lipopolysaccharide-induced airway neutrophilia: IL-15 as a possible trigger. JImmunol (2003) 170(4):2106-12. doi:10.4049/jimmunol.170.4.2106

16. Griffin GK, Newton G, Tarrio ML, Bu DX, Maganto-Garcia E, Azcutia V, et al. IL-17 and TNF-alpha sustain neutrophil recruitment during inflammation through synergistic effects on endothelial activation. JImmunol (2012) 188(12):6287-99. doi:10.4049/jimmunol.1200385

17. Bisgaard H, Hermansen MN, Buchvald F, Loland L, Halkjaer LB, Bonnelykke K, et al. Childhood asthma after bacterial colonization of the airway in neonates. N Engl J Med (2007) 357(15):1487-95. doi:10.1056/ NEJMoa052632

18. Bonnelykke K, Vissing NH, Sevelsted A, Johnston SL, Bisgaard H. Association between respiratory infections in early life and later asthma is independent of virus type. J Allergy Clin Immunol (2015) 136(1):81-6.e4. doi:10.1016/j. jaci.2015.02.024

19. Curtis MM, Way SS. Interleukin-17 in host defence against bacterial, mycobacterial and fungal pathogens. Immunology (2009) 126(2):177-85. doi:10.1111/j.1365-2567.2008.03017.x

20. Zhang Z, Clarke TB, Weiser JN. Cellular effectors mediating Th17-dependent clearance of pneumococcal colonization in mice. JClin Invest (2009) 119(7):1899-909. doi:10.1172/JCI36731

21. Jatakanon A, Uasuf C, Maziak W, Lim S, Chung KF, Barnes PJ. Neutrophilic inflammation in severe persistent asthma. Am J Respir Crit Care Med (1999) 160(5):1532-9. doi:10.1164/ajrccm.160.5.9806170

22. Green RH, Brightling CE, Woltmann G, Parker D, Wardlaw AJ, Pavord ID. Analysis of induced sputum in adults with asthma: identification of subgroup with isolated sputum neutrophilia and poor response to inhaled corticosteroids. Thorax (2002) 57(10):875-9. doi:10.1136/thorax.57.10.875

23. McKinley L, Alcorn JF, Peterson A, DuPont RB, Kapadia S, Logar A, et al. $\mathrm{T}(\mathrm{H}) 17$ cells mediate steroid-resistant airway inflammation and airway hyperresponsiveness in mice. J Immunol (2008) 181(6):4089-97. doi:10.4049/ jimmunol.181.6.4089
24. Chu DK, Al-Garawi A, Llop-Guevara A, Pillai RA, Radford K, Shen P, et al. Therapeutic potential of anti-IL-6 therapies for granulocytic airway inflammation in asthma. Allergy Asthma Clin Immunol (2015) 11(1):14. doi:10.1186/ s13223-015-0081-1

25. Rincon M, Irvin CG. Role of IL-6 in asthma and other inflammatory pulmonary diseases. Int J Biol Sci (2012) 8(9):1281-90. doi:10.7150/ ijbs. 4874

26. Scheller J, Chalaris A, Schmidt-Arras D, Rose-John S. The pro- and anti-inflammatory properties of the cytokine interleukin-6. Biochim Biophys Acta (2011) 1813(5):878-88. doi:10.1016/j.bbamcr.2011.01.034

27. Dejager L, Dendoncker K, Eggermont M, Souffriau J, Van Hauwermeiren F, Willart M, et al. Neutralizing TNF alpha restores glucocorticoid sensitivity in a mouse model of neutrophilic airway inflammation. Mucosal Immunol (2015) 8(6):1212-25. doi:10.1038/mi.2015.12

28. Berry MA, Hargadon B, Shelley M, Parker D, Shaw DE, Green RH, et al. Evidence of a role of tumor necrosis factor alpha in refractory asthma. $N$ Engl $J$ Med (2006) 354(7):697-708. doi:10.1056/NEJMoa050580

29. Lukacs NW, Strieter RM, Chensue SW, Widmer M, Kunkel SL. TNF-alpha mediates recruitment of neutrophils and eosinophils during airway inflammation. J Immunol (1995) 154(10):5411-7.

30. Scheurich P, Thoma B, Ucer U, Pfizenmaier K. Immunoregulatory activity of recombinant human-tumor necrosis factor (TNF)-alpha - induction of TNF receptors on human T-cells and TNF-alpha-mediated enhancement of T-cell responses. J Immunol (1987) 138(6):1786-90.

31. Sullivan DE, Ferris MB, Pociask D, Brody AR. Tumor necrosis factor-alpha induces transforming growth factor-alpha expression in lung fibroblasts through the extracellular signal-regulated kinase pathway. Am J Respir Cell Mol Biol (2005) 32(4):342-9. doi:10.1165/rcmb.2004-0288OC

32. Iyoda M, Shibata T, Kawaguchi M, Hizawa N, Yamaoka T, Kokubu F, et al. IL-17A and IL-17F stimulate chemokines via MAPK pathways (ERK1/2 and p38 but not JNK) in mouse cultured mesangial cells: synergy with TNF-alpha and IL-1beta. Am J Physiol Renal Physiol (2010) 298(3):F779-87. doi:10.1152/ ajprenal.00198.2009

33. Nonaka M, Ogihara N, Fukumoto A, Sakanushi A, Kusama K, Pawankar R, et al. Synergistic induction of macrophage inflammatory protein-3alpha;/ CCL20 production by interleukin-17A and tumor necrosis factor-alpha; in nasal polyp fibroblasts. World Allergy Organ J (2009) 2(10):218-23. doi:10.1097/WOX.0b013e3181bdd219

34. Wenzel SE, Barnes PJ, Bleecker ER, Bousquet J, Busse W, Dahlen SE, et al. A randomized, double-blind, placebo-controlled study of tumor necrosis factor-alpha blockade in severe persistent asthma. Am J Respir Crit Care Med (2009) 179(7):549-58. doi:10.1164/rccm.200809-1512OC

35. Erin EM, Leaker BR, Nicholson GC, Tan AJ, Green LM, Neighbour H, et al. The effects of a monoclonal antibody directed against tumor necrosis factor-alpha in asthma. Am J Respir Crit Care Med (2006) 174(7):753-62. doi:10.1164/rccm.200601-072OC

36. Foster PS, Ming Y, Matthei KI, Young IG, Temelkovski J, Kumar RK. Dissociation of inflammatory and epithelial responses in a murine model of chronic asthma. Lab Invest (2000) 80(5):655-62.

Conflict of Interest Statement: The authors declare that the research was conducted in the absence of any commercial or financial relationships that could be construed as a potential conflict of interest.

Copyright $\odot 2017$ Alnahas, Hagner, Raifer, Kilic, Gasteiger, Mutters, Hellhund, Prinz, Pinkenburg, Visekruna, Garn and Steinhoff. This is an open-access article distributed under the terms of the Creative Commons Attribution License (CC BY). The use, distribution or reproduction in other forums is permitted, provided the original author(s) or licensor are credited and that the original publication in this journal is cited, in accordance with accepted academic practice. No use, distribution or reproduction is permitted which does not comply with these terms. 